\title{
Avanços na Síntese Química: Aquecimento Óhmico
}

\author{
Vera L.M. Silva, ${ }^{1, *}$ Ana M.G. Silva, ${ }^{2}$ Luís M.N.B.F. Santos ${ }^{3}$ e Artur M.S. Silva ${ }^{1}$ \\ ${ }^{1}$ QOPNA, Departamento de Química, Universidade de Aveiro, 3810-193 Aveiro, Portugal \\ ${ }^{2}$ UCIBIO / REQUIMTE, Departamento de Química e Bioquímica, Faculdade de Ciências, Universidade do Porto, $4169-007$ Porto, Portugal \\ ${ }^{3}$ CIQUP, Departamento de Química e Bioquímica, Faculdade de Ciências da Universidade do Porto, 4169-007 Porto, Portugal
} verasilva@ua.pt

\begin{abstract}
Advances in Chemical Synthesis: Ohmic Heating - The ohmic heating, also known as direct Joule heating, is a method of thermal processing of materials mainly used in the food industry. Its use in organic synthesis, in the heating of chemical reactors, is an emerging method that shows great potential and whose development started recently in Portugal. Herein it is presented and explained the fundamentals of ohmic heating and made a qualitative and quantitative comparison with other common heating methods. It is also shown the state-of-the-art and future perspectives of the area of study of ohmic heating. A brief description of the ohmic reactor prototype in operation is presented, as well as specific examples of the application of this reactor in organic synthesis at laboratory scale. The advantages and limitations of this new heating technology are also stated.
\end{abstract}

O aquecimento óhmico, ou aquecimento direto por efeito de Joule, é um método de processamento térmico de materiais usado principalmente na indústria alimentar. A sua utilização em síntese orgânica, no aquecimento de reatores químicos, é um método emergente que apresenta grandes potencialidades e cujo desenvolvimento teve início muito recentemente em Portugal. Neste artigo são apresentados e explicados os fundamentos do aquecimento óhmico e é feita a comparação qualitativa e quantitativa com outros métodos comuns de aquecimento. Pretende-se ainda dar a conhecer o estado de desenvolvimento e perspetivas futuras da área de estudo do aquecimento óhmico. É feita a descrição sumária do prótotipo do reator óhmico que está em funcionamento e são apresentados exemplos da sua aplicação em síntese orgânica à escala laboratorial bem como as vantagens e limitações inerentes a esta nova tecnologia de aquecimento.

\section{INTRODUÇÃO}

Nas últimas décadas, as tecnologias de aquecimento têm registado avanços muito significativos com o desenvolvimento de novas metodologias como o aquecimento óhmico (AO), o aquecimento usando radiação micro-ondas (MO) e o aquecimento indutivo (AI). Todos esses métodos avançados de aquecimento são em determinadas situações energeticamente mais eficientes e conduzem a uma perturbação térmica diferenciada relativamente ao aquecimento convencional (AC), já que o calor é gerado internamente no meio a aquecer.

As primeiras descrições de processos realizados com recurso ao AO surgiram no final do século XIX, quando se descreveu a pasteurização do leite por bombeamento do fluído entre duas placas metálicas (elétrodos), às quais foi aplicada uma diferença de potencial (Figura 1) [1]. Nessa altura surgiram outras aplicações do AO na indústria alimentar, nomeadamente na cozedura de sanduíches tipo Frankfurter [2] e no branqueamento de batatas [3]. Nos anos que se seguiram, a tecnologia praticamente desapareceu devido à falta de controlo dos processos e ao uso inadequado de elétrodos constituídos por materiais não inertes [4]. Pensava-se que a passagem de corrente elétrica pelos alimentos poderia trazer efeitos colaterais e até letais aos consumidores. No entanto, nas últimas duas décadas a técnica voltou a renascer, muito graças ao aparecimento de novos materiais utilizados na construção dos elétrodos e ao desenho de reatores mais eficientes e versáteis. O conselho inglês de eletricidade patenteou um sistema de AO em contínuo e licenciou a tecnologia à APV Baker [5]. Desde então, o AO emergiu como método avançado de aquecimento com aplicações nas indústrias alimentar, química, farmacêutica e agroquímica.

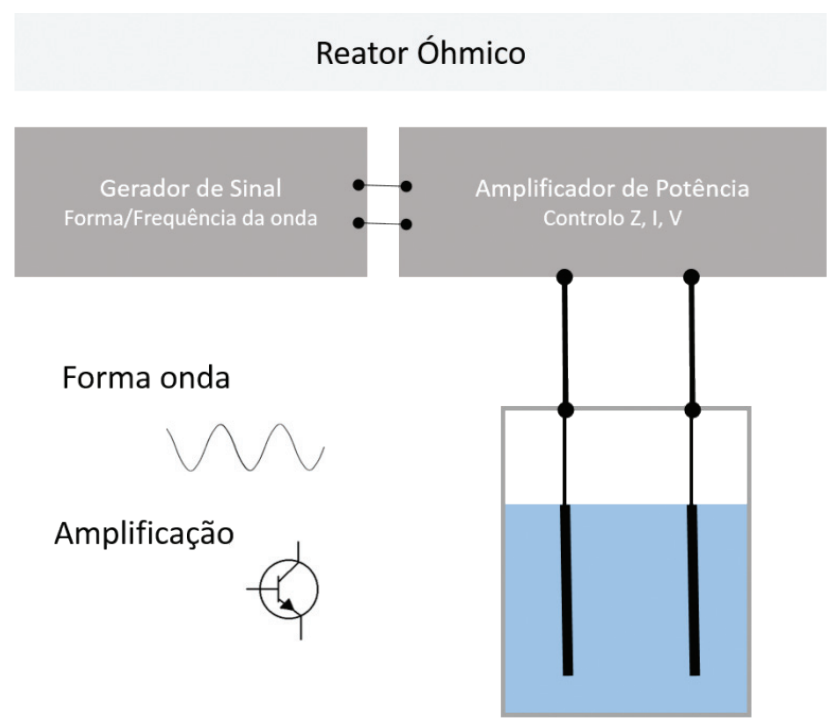

Figura 1 - Princípios de funcionamento do aquecimento óhmico.

\section{Princííos do Aquecimento Óhmico}

O AO, também designado como aquecimento Joule, aquecimento por resistência elétrica ou aquecimento electrocondutivo, é definido como um processo no qual o material (ou a mistura reacional), que funciona como uma resistên- 
cia elétrica, é aquecido pela passagem direta de corrente elétrica alternada. A energia elétrica é dissipada diretamente por efeito de Joule no meio, sendo o calor gerado com uma eficiência energética muito elevada, o que resulta num aquecimento rápido e uniforme (temperatura homogénea) e ainda num aumento considerável da dinâmica molecular do meio reacional. O AO distingue-se dos outros métodos de aquecimento por envolver o uso de elétrodos que estão em contacto com o meio a aquecer, ao contrário do aquecimento com MO (uso de radiação com frequência de 2,45 $\mathrm{GHz}$ ) [6] e do AI (uso de nanopartículas magnéticas) [7] nos quais os elétrodos estão ausentes. A frequência aplicada é variável e inferior às frequências rádio e micro-ondas. A forma de onda pode ser variável, sendo mais frequente o uso da onda sinusoidal (Tabela 1).

Tabela 1 - Principais tipos de aquecimento com geração interna de calor no meio reacional.

\begin{tabular}{|c|c|c|}
\hline \multicolumn{3}{|c|}{ Tipos de aquecimento } \\
\hline Micro-ondas, MO
\end{tabular}

Existem vários fatores conhecidos por influenciarem a eficiência do aquecimento óhmico, tais como:

(i) A condutividade elétrica. Considera-se uma substância como condutora elétrica se neste material existir uma elevada mobilidade dos transportadores de carga elétrica. Este fluxo de carga elétrica num meio é conhecido como eletricidade. A eletricidade pode ser descrita de forma simples pela corrente elétrica, voltagem (ou tensão) e impedância do meio. A condutividade elétrica $(\sigma)$ é uma medida da eficácia de um meio transferir carga elétrica, expressa em Siemens por metro $(\mathrm{S} / \mathrm{m})$, e é a razão entre a densidade de corrente e a força do campo elétrico sendo afetada pelas caraterísticas do meio, concentração de espécies carregadas ou de elevada polarizabilidade. Na terminologia do AO, a condutividade é uma medida do conteúdo iónico. Para substâncias alimentares, o aditivo iónico mais comum é o cloreto de sódio $(\mathrm{NaCl})$. Quanto maior for a quantidade de sais dissolvidos numa substância, maior será a condutividade [8].

(ii) Frequência e forma de onda. Por razões práticas as frequências mais utilizadas no AO industrial são 50 e $60 \mathrm{~Hz}$ (frequência da rede). A frequência e a forma de onda aplicada afetam os valores da eficiência e qualidade do processo de AO dada a usual elevada dependência da impedância relativamente à frequência aplicada (fenómenos capacitivos e indutivos) [9].

(iii) A intensidade de corrente elétrica. É medida em amperes, em que 1 Ampére é equivalente ao fluxo de uma carga igual à carga transportada por $\sim 6 \times 10^{18}$ eletrões por segundo num meio condutor [10].

(iv) A voltagem (ou tensão). É a pressão dos eletrões, ou seja, é a medida da capacidade de movimentar uma carga elétrica através de uma resistência [10] e pode ser calculada através da intensidade de corrente e da resistência do material [V = R x I (em que V = voltagem; $\mathrm{I}$ = intensidade de corrente; $\mathrm{R}$ = resistência)]. Este princípio é conhecido como a lei de Ohm, publicado pela primeira vez pelo físico Georg Ohm em 1827 e refere que: "a intensidade de corrente elétrica que percorre um condutor é diretamente proporcional à diferença de potencial (ou seja, à voltagem) e inversamente proporcional à resistência do circuito”. A lei de Ohm é geralmente aplicada a circuitos de corrente contínua (DC), mas pode ser também aplicada a circuitos de corrente alternada (AC), aplicando a fórmula:

$\mathrm{V}_{\mathrm{m}}=\mathrm{Z} \times \mathrm{I}_{\mathrm{m}}$

(Equação 1)

em que $\mathbf{V}_{\mathbf{m}}$ é a voltagem de pico do gerador de corrente elétrica AC aplicada no circuito; $\mathbf{I}_{\mathrm{m}}$ é a intensidade de corrente elétrica de pico do circuito e $\mathbf{Z}$ é a impedância do circuito (ou seja, a resistência do circuito à passagem de corrente elétrica).

(v) Potência média dissipada (efeito de Joule). Da resistência elétrica resulta uma força no sentido oposto à deslocação da carga elétrica. Esta força origina a realização de trabalho, transferência de energia no sistema. A energia transferida é dissipada no sistema sob a forma de energia térmica. A lei física que relaciona a energia térmica dissipada por uma corrente elétrica que percorre determinado condutor num determinado tempo é conhecida como lei de Joule (também conhecida como efeito Joule) e pode ser enunciada pela fórmula:

$\mathrm{P}_{\mathrm{rms}}=\mathrm{I}_{\mathrm{mq}} \times \mathrm{V}_{\mathrm{mq}}$

(Equação 2)

em que $\mathbf{P}_{\text {rms }}$ é a potência média dissipada numa resistência com uma corrente alternada, $\mathbf{I}_{\mathbf{m q}}$ é a corrente média quadrática no sistema e $\mathbf{V}_{\mathbf{m q}}$ é a voltagem média quadrática aplicada ao sistema. Assim, num circuito em AC, usando uma onda sinusoidal, tem-se que:

$\mathrm{I}_{\mathrm{mq}}=\mathrm{I}_{\mathrm{m}} \times 2^{-1 / 2}$

(Equação 3)

$\mathrm{V}_{\mathrm{mq}}=\mathrm{V}_{\mathrm{m}} \times 2^{-1 / 2}$

(Equação 4)

Através de dedução matemática e aplicando o enunciado da lei de Ohm (Equação 1) ao enunciado da lei de Joule (Equação 2) [e considerando que a condutividade elétrica $(\sigma, \mathrm{S} / \mathrm{m})$ relaciona-se com a impedância do sistema de forma a que, quando multiplicadas as suas grandezas, obtém-se resultado igual a um $(\sigma \times \mathrm{Z}=1)$ ], pode-se relacionar a energia dissipada pela corrente elétrica com a condutivida- 
de elétrica, ou seja, a energia dissipada pela corrente elétrica, num circuito elétrico, é proporcional à condutividade elétrica do circuito e ao quadrado do potencial aplicado no mesmo (Equação 5) [10].

$\mathrm{P}_{\mathrm{rms}}=\mathrm{I}_{\mathrm{mq}} \times \mathrm{V}_{\mathrm{mq}}=\mathrm{V}_{\mathrm{mq}}{ }^{2} \times \mathrm{Z}^{-1}=\sigma \times \mathrm{V}_{\mathrm{mq}}^{2}$

(Equação 5)

Estes conceitos permitem compreender melhor o tipo de dados que se podem medir ou monitorizar no AO e como estes se podem relacionar.

\section{INSTRUMENTAÇÃO}

No que diz respeito aos sistemas de AO existem inúmeras possibilidades para a conceção de um sistema deste tipo. Contudo, há vários componentes e caraterísticas que todos devem apresentar:

(i) Fonte de alimentação (+ gerador de sinal): para fornecer a corrente elétrica alternada com as caraterísticas pretendidas;

(ii) Elétrodos: conectados à fonte de alimentação e que devem estar em contacto físico com a substância ou meio a aquecer, a fim de fazer passar a corrente elétrica. A distância entre os elétrodos e a sua forma podem variar dependendo do tamanho do sistema, mas variando esta distância, a força do campo elétrico, expressa em volt por centímetro [V/cm], varia também. Podem ser usados diferentes materiais para a construção dos elétrodos, sendo mais comuns o aço inox ou o titânio. As reações eletroquímicas na interface elétrodo/solução, altamente indesejáveis, são evitadas recorrendo à utilização de uma elevada frequência;

(iii) Sensor de temperatura: como por exemplo, um termopar eletricamente isolado;

(iv) Data logger e computador: para aquisição de dados e registar as informações essenciais, tais como a temperatura, a voltagem (tensão), a intensidade da corrente e a potência ao longo do tempo.

Como no AO a energia térmica é gerada no interior do meio a aquecer, teoricamente, não existe limite superior para a temperatura que pode ser produzida. No entanto, vários fatores influenciam a temperatura alcançada pelo sistema, tais como: i) a condutividade elétrica do meio; ii) a conceção do sistema; iii) o tempo que o meio é submetido ao aquecimento; iv) as propriedades termofísicas do meio; v) a intensidade do campo elétrico; e vi) a dependência da impedância do meio com a temperatura [11].

Atualmente os sistemas de AO estão a ser especificamente concebidos para trabalhar em fluxo contínuo. Em oposição aos sistemas tradicionais que operam em vasos reacionais (balões de fundo redondo ou vasos fechados), os sistemas em fluxo contínuo tipicamente operam em microrreatores de dimensões muito reduzidas, onde as reações são realizadas em condições rigorosamente controladas num espaço muito confinado. A grande inovação dos sistemas em fluxo contínuo é a extraordinária eficiência de aquecimento e transferência de massa, que permite aumentar a velocidade da reação e consequentemente a produtividade do processo [12].

No desenho e conceção de sistemas com AO em fluxo contínuo, podem ser consideradas duas geometrias distintas que são também as mais comuns nestes sistemas (Tabela 2):

(i) Configuração transversal: o produto é bombeado no sentido dos elétrodos e perpendicular ao campo elétrico. Este arranjo é mais adequado para fluidos de baixa condutividade $(<5 \mathrm{~S} / \mathrm{m})$ e não contendo partículas sólidas;

(ii) Configuração colinear: o produto é bombeado de um elétrodo para o outro e o fluxo é paralelo ao campo elétrico. A configuração colinear é montada a partir de duas unidades - o compartimento dos elétrodos e o tubo espaçador. Ao juntar-se alternadamente o compartimento dos elétrodos / tubo espaçador / compartimento dos elétrodos, chega-se progressivamente a sistemas de potência mais elevada.

Tabela 2 - Configuração transversal versus configuração colinear.

\begin{tabular}{|c|c|}
\hline Configuração transversal & Configuração colinear \\
\hline &
\end{tabular}

\section{Vantagens do Aquecimento Óhmico}

O AO é hoje utilizado principalmente pela indústria alimentar e o processamento óhmico permite o aquecimento de materiais com velocidades elevadas (de alguns segundos a alguns minutos) [13]. Também permite, em certas circunstâncias, aquecer partículas e fluídos veiculares a taxas comparáveis, tornando assim possível a utilização de técnicas de alta temperatura em curto espaço de tempo (HTST) e ultra-alta temperatura (UHT), em sólidos ou em materiais em suspensão, aumentando a qualidade do produto final e por isso acrescentando-lhe valor [14,15]. Este cenário muito positivo e desejável dificilmente é conseguido usando o AC [16].

Como principais vantagens do AO destacam-se:

- O processamento contínuo, sem a existência de superfícies de transferência de calor;

- O aquecimento rápido e uniforme (temperatura homogénea) de fases líquidas e sólidas com o mínimo de danos e perdas de nutrientes provocados pelo aqueci- 
mento (ao contrário do aquecimento com MO, que tem uma profundidade de penetração limitada nos materiais sólidos); por exemplo, temperaturas elevadas para processamento UHT podem ser rapidamente atingidas;

- Apresenta-se como um processo ideal para materiais sensíveis à deformação devido à baixa velocidade de fluxo utilizada;

- Otimização do capital de investimento e da segurança dos produtos como resultado da elevada eficiência do processamento;

- Redução da incrustação das substâncias a aquecer, quando comparada com o AC, e consequentemente redução dos requisitos de limpeza; apresenta ainda baixo risco de danos ao produto devido à queima;

- Melhor controlo do processo, com custos de manutenção reduzidos;

- Sistema mais “amigo” do meio ambiente;

- Manutenção da cor e do valor nutritivo dos alimentos;

- Aquecimento de alimentos com partículas e com misturas líquido/partículas;

- Alta eficiência de conversão de energia.

A aplicação do AO em síntese química é ainda um conceito emergente, contudo já foram demonstradas algumas vantagens do AO em comparação com o AC e aquecimento com MO, nomeadamente:

- Redução dos tempos de reação;

- Melhores rendimentos e melhores seletividades;

- Baixa inércia térmica;

- Maior facilidade de escalamento do processo para a escala piloto ou mesmo até para a escala industrial quando comparado com o MO.

\section{Desvantagens do Aquecimento Óhmico}

Allen e colaboradores [17] compararam os custos de instalação e operação de um sistema de processamento com o AO com o AC, e concluíram que o AO revelou ser mais dispendioso do que os métodos convencionais de processamento. Por outro lado, os alimentos que contêm glóbulos de gordura não são eficientemente aquecidos no AO porque não são condutores, devido à falta de água e sal na sua matriz. Se esses glóbulos estiverem presentes numa região de elevada condutividade elétrica, onde há passagem de corrente elétrica, eles podem aquecer lentamente por transferência de energia. Qualquer agente patogénico que esteja presente nesses glóbulos irá receber um tratamento térmico inferior ao restante alimento [18].

No que se refere à aplicação do AO em síntese química as principais desvantagens são:

- O meio a aquecer tem de ser condutor, contudo a aplicação do AO em meios reacionais com baixa impedância, ou seja, alta condutividade, poderá ser mais problemática;
- A falta de conhecimento e informação mais generalizada sobre o processo;

- Necessidade de pesquisa e desenvolvimento adicionais para entender e validar plenamente esta nova tecnologia no que diz respeito ao seu desempenho e segurança quando aplicado a transformações químicas.

\section{Síntese Assistida por Aquecimento Óhmico em Portugal}

Uma pesquisa na Scopus database usando as palavras-chaves "Ohmic" e "heating” revela que em Portugal, nos últimos 10 anos (2006-2016) foram publicados 30 artigos científicos em revistas internacionais envolvendo o estudo/ aplicação do AO nas áreas da física e astronomia, agricultura e biologia, química, engenharia química e bioquímica. Importa realçar que, nos últimos três anos há uma tendência crescente no número de artigos publicados, revelador do crescente interesse da comunidade científica por este processo de aquecimento (Figura 2) [19].

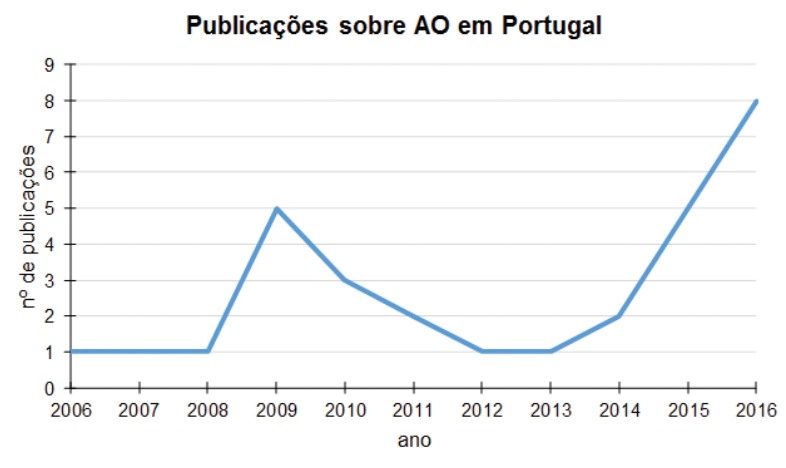

Figura 2 - Evolução do número de artigos sobre AO publicados por ano no período 2006-2016, em Portugal.

Na química e áreas mais diretamente relacionadas com a química, os artigos publicados neste período são, na sua maioria, estudos sobre a utilização do AO como um método alternativo de processamento de alimentos e na inativação de microrganismos. Em muitos destes estudos estiveram envolvidos investigadores do Centro de Engenharia Biológica da Universidade do Minho que têm dado um contributo importante nesta área [20-27].

Só muito recentemente, em 2013, surgiu uma publicação sobre a utilização do AO em síntese química, mais concretamente em síntese orgânica [28]. Tanto quanto sabemos, este estudo é pioneiro uma vez que até essa data ainda não existia nenhum reator de AO para síntese química à escala laboratorial e a utilização deste método de aquecimento para este fim ainda não tinha sido reportada na literatura.

Investigadores da Unidade de Investigação QOPNA do Departamento de Química da Universidade de Aveiro (QOPNA/UA), em colaboração com investigadores da Faculdade de Ciências da Universidade do Porto (CIQUP e UCIBIO/REQUIMTE) e da Universidade do Minho (CEB/ UM) desenharam, construíram e patentearam o primeiro reator com AO para síntese química à escala laboratorial $[28,29]$ (Figura 3). 


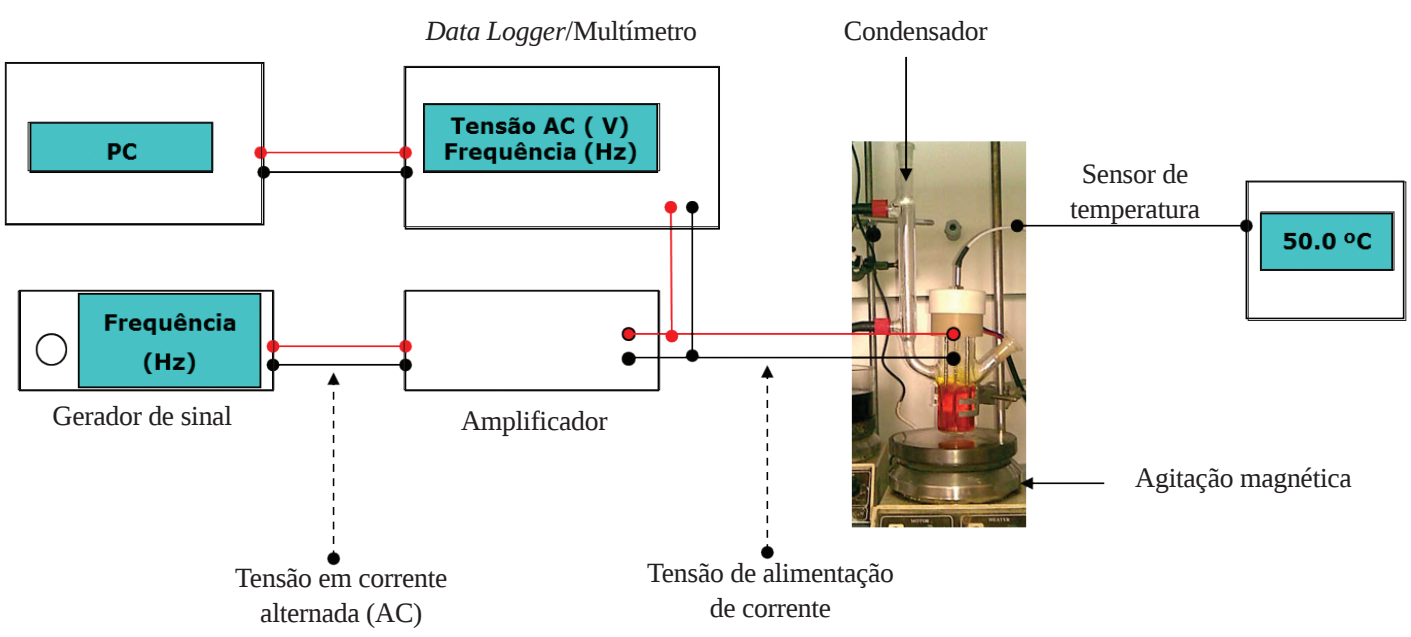

Figura 3 - Representação esquemática do reator de AO para síntese química e sistemas periféricos (Patente Portuguesa nº 105908).

Usando este reator foram efetuadas as primeiras experiências de síntese orgânica. Foram selecionadas quatro reações químicas consideradas representativas em síntese orgânica, uma reação de Diels-Alder, uma substituição nucleofílica, uma $N$-alquilação e uma reação de acoplamento cruzado carbono-carbono (reação de Suzuki-Miyaura) e essas reações foram efetuadas em meio aquoso usando o AO, MO e AC de modo a comparar os três processos de aquecimento. Este estudo foi publicado em 2013 e demonstrou que o $\mathrm{AO}$ permite um aquecimento rápido e uniforme do meio reacional e aumenta a dinâmica/mobilidade das partículas carregadas em solução o que conduz a melhores rendimentos e tempos de reação mais curtos. Foi possível concluir que o AO pode ser usado como uma alternativa ao MO uma vez que os resultados obtidos são semelhantes e em alguns casos até foram superiores aos resultados obtidos em MO [28].

Desde então, estes investigadores têm vindo a desenvolver novas metodologias de síntese, mais sustentáveis, baseadas no reator de AO. Em 2015 foi publicada a síntese de uma quimioteca de 3 -arilquinolin-4(1H)-onas 3 que foram sintetizadas no reator de AO através de uma reação de acoplamento cruzado de Suzuki-Miyaura de 3-iodoquinolin-4(1H)-onas 1 com diferentes ácidos borónicos 2 (Figura 4) [30]. Foi desenvolvido um protocolo simples e eficiente para esta reação, usando água como solvente, na presença de uma base inorgânica, de um catalisador de transferência de fase, o TBAB (brometo de tetrabutilamónio) e de um catalisador de paládio(II), o $\mathrm{Pd}(\mathrm{OAc})_{2}$, na ausência de ligando. A possibilidade de usar diferentes ácidos borónicos, contendo substituintes dadores e/ou sacadores de eletrões,<smiles>[R]n1cc(I)c(=O)c2ccccc21</smiles>

1

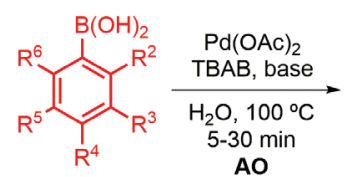

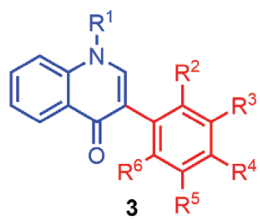

(12 exemplos)
$\mathrm{R}^{1}=\mathrm{CH}_{3}$, pentil; $\mathrm{R}^{2}=\mathrm{R}^{5}=\mathrm{R}^{6}=\mathrm{H}, \mathrm{CH}_{3}$;

$\mathrm{R}^{3}=\mathrm{H}, \mathrm{OCH}_{3}, \mathrm{CHO} ; \mathrm{R}^{4}=\mathrm{H}, \mathrm{OCH}_{3}, \mathrm{OH}, \mathrm{N}\left(\mathrm{CH}_{3}\right)_{2}, \mathrm{CHO}, \mathrm{NO}_{2}$

Figura 4 - Síntese de 3-arilquinolin-4(1H)-onas (3) usando aquecimento óhmico. a facilidade de execução e curto tempo de reação e os bons rendimentos obtidos tornam este protocolo uma opção viável e vantajosa para a preparação de 3-arilquinolin-4(1H)-onas. Foi demonstrado que após um simples workup da reação é possível recuperar não só o catalisador de paládio, como o sistema composto pelo catalisador de paládio/ água/TBAB podendo ser reutilizados durante pelo menos sete ciclos consecutivos sem perda significativa de atividade, tornando este método ainda mais vantajoso do ponto de vista económico e ambiental [30].

Em 2016 foi publicada a síntese de (E)-3-estirilquinolin-4(1H)-onas 5 em AO, através da reação de acoplamento cruzado de Mizoroki-Heck (mais vulgarmente conhecida por reação de Heck) de 3-iodoquinolin-4(1H)-onas 1 com estirenos substituídos 4, em água em condições de transferência de fase (Figura 5), usando um protocolo semelhante ao da reação de Suzuki apresentada na Figura 4 [31]. Também foram usados acrilatos em vez de estirenos dando origem a novas quinolin-4(1H)-onas com diferentes substituintes na posição 3 [31]. A comparação desta metodologia com outras rotas de síntese de (E)-3-estirilquinolin-4(1H)-onas, em que a reação de Heck é efetuada usando solventes orgânicos, na presença de ligandos de fosfina, em AC ou em MO, revelou as vantagens da utilização deste protocolo e do AO na síntese destes compostos, que foram obtidos em melhores rendimentos e em tempos de reação mais curtos do que em AC e em MO [31].

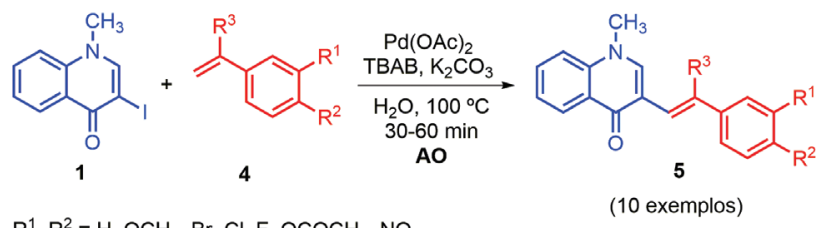

$\mathbf{R}^{1}, \mathbf{R}^{2}=\mathrm{H}, \mathrm{OCH}_{3}, \mathrm{Br}, \mathrm{Cl}, \mathrm{F}, \mathrm{OCOCH}_{3}, \mathrm{NO}_{2}$

(10 exemplos) $\mathrm{R}^{3}=\mathrm{H}, \mathrm{CH}_{3}$

Figura 5 - Síntese de (E)-3-estirilquinolin-4(1H)-onas (5) usando aquecimento óhmico.

Recentemente o AO foi usado na síntese de novos derivados de cumarinilporfirinas contendo unidades do tipo pirano[3,2-c]cumarina numa posição $\beta$-pirrólica do macrociclo porfirínico. Estes compostos foram obtidos por uma estratégia sintética que envolve uma reação de Kno- 
evenagel seguida de uma reação hetero-Diels-Alder entre o 2-vinil-5,10,15,20-tetrafenilporfirinatozinco(II) (9) e 3-(arilmetileno)cromano-2,4-dionas (8) geradas in situ por reação da 4-hidroxicumarina (6) com diferentes aldeídos aromáticos (7) em meio aquoso (Figura 6). Os resultados obtidos em AO foram comparados com os resultados obtidos em AC usando água ou dioxano como solvente [32].

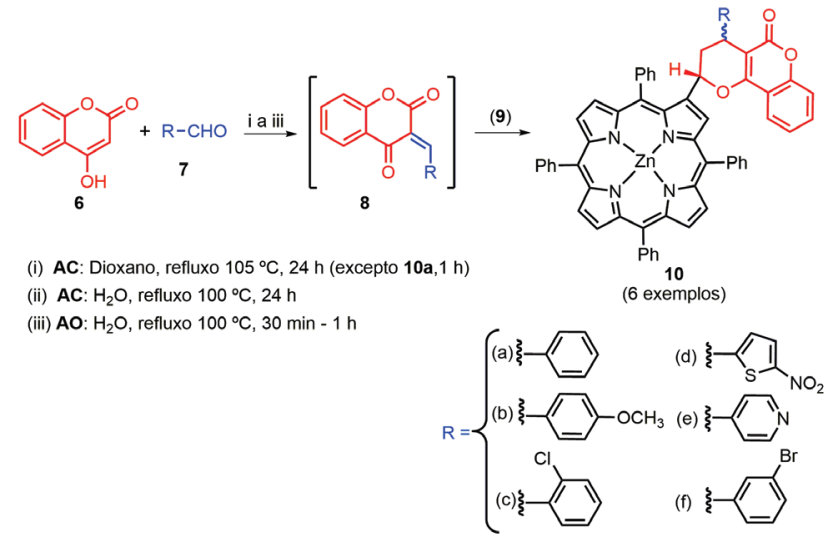

Figura 6 - Síntese de cumarinilporfirinas usando aquecimento óhmico.

A reação origina uma mistura diastereomérica de cumarinilporfirinas $\mathbf{1 0}$ sendo mais eficiente em AO, uma vez que ocorre mais rapidamente, com melhor diastereosseletividade e com maior economia atómica, uma vez que não é necessária uma segunda adição de aldeído e de cumarina, como acontece em AC (Figura 7) [32].

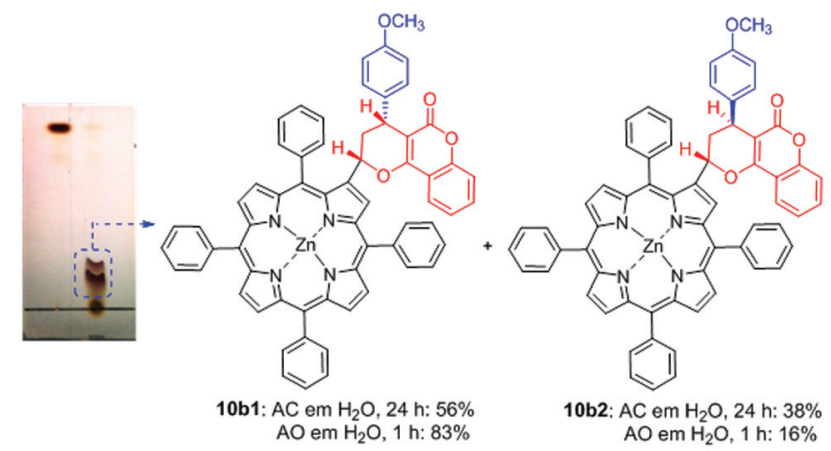

Figura 7 - Comparação dos resultados obtidos em AC e em AO na síntese da cumarinilporfirina $\mathbf{1 0 b}$.

Os líquidos iónicos são excelentes solventes para reações em AO uma vez que são naturalmente condutores, além de serem praticamente não voláteis, não inflamáveis e reutilizáveis, constituindo uma alternativa ao uso de solventes orgânicos, que acarretam riscos associados à sua toxicidade, volatilidade e inflamabilidade. A utilização de líquidos iónicos, em particular do [bmim] Br (brometo de 1-butil-3-metilimidazólio) em reações de síntese de derivados 1-haloalceno promovidas por índio (In) em AO (Figura 8), constitui uma alternativa mais verde e eficiente para a síntese deste tipo de compostos [33]. A utilização do AO neste tipo de reações permitiu uma redução significativa do tempo de reação e uma melhoria da seletividade, como se pode ver no exemplo apresentado na Figura 9. Nestas reações o aquecimento com MO não foi tão eficiente como o AO. Em AO o 1-bromoalceno 12a foi obtido com um rendimento de $84 \%$ e diastereosseletividade de 90:10 E/Z (entrada 4), enquanto que em MO o rendimento foi de $46 \%$ e a diastereosseletividade de 61:39 E/Z (entrada 5) [33]. (a)<smiles>[R]C=C(Br)Br</smiles>

11

(b)

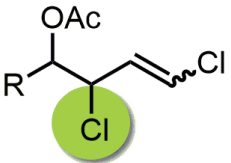

13

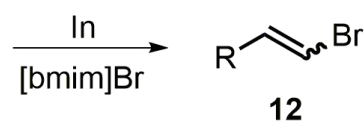

(17 exemplos)

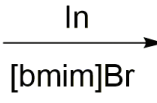<smiles>[R]C=CC=CCl</smiles>

14

(10 exemplos)
$\mathrm{R}=$ arilo, alquilo, açúcar, $4 H$-cromen-4-ona

Figura 8 - Síntese de 1-haloalcenos promovida por índio usando aquecimento óhmico.

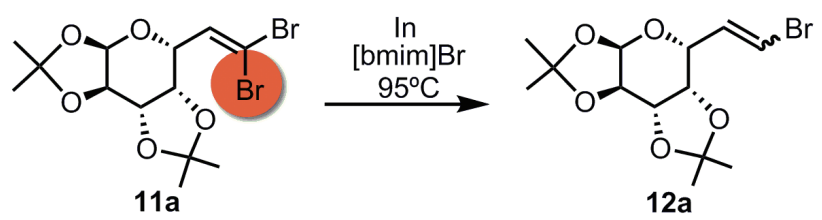

\begin{tabular}{|c|c|c|c|c|}
\hline Entrada & $\begin{array}{c}\text { Método de } \\
\text { aquecimento }\end{array}$ & $\mathbf{t}$ (h) & $\begin{array}{c}\text { Rendimento } \\
(\mathbf{\%})\end{array}$ & $\boldsymbol{E} / \mathbf{Z}$ \\
\hline 1 & AC & 12 & 45 & $92 / 8$ \\
\hline 2 & AC & $\mathbf{3 6}$ & $\mathbf{8 2}$ & $\mathbf{9 0 / 1 0}$ \\
\hline 3 & AO & 1 & 46 & $91 / 9$ \\
\hline 4 & AO & $\mathbf{3}$ & $\mathbf{8 4}$ & $\mathbf{9 0 / 1 0}$ \\
\hline 5 & MO & $\mathbf{3}$ & $\mathbf{4 6}$ & $\mathbf{6 1 / 3 9}$ \\
\hline
\end{tabular}

Figura 9 - Comparação dos rendimentos e seletividades obtidos na síntese do 1-bromoalceno 12a em AC, MO e AO.

\section{Síntese Assistida por Aquecimento Óhmico: Que FUTURO?}

A utilização do AO para síntese química e em particular na síntese orgânica é um conceito ainda muito recente. No entanto, os estudos já efetuados mostram que este método de aquecimento tem grandes potencialidades para este fim e que há ainda muito trabalho a desenvolver e uma margem de aperfeiçoamento muito grande relativamente a outros métodos de aquecimento. Por um lado, no que respeita ao reator de $\mathrm{AO}$, os objetivos futuros passam pela sua otimização e miniaturização de modo a construir um equipamento mais adequado para uso numa hotte, pela construção de vasos reacionais que possam trabalhar sob pressão (vaso fechado), pelo acoplamento do AO com outras técnicas como, por exemplo, ultrassons (US) assim como a construção de reatores de AO para operar em modo contínuo (flow). Estas são várias possibilidades a explorar no que respeita à construção de novos reatores. Por outro lado, apesar de a inércia térmica neste método de aquecimento ser muito baixa relativamente a outros métodos e empiricamente ser fácil de compreender que se trata de um método energeticamente mais eficiente, seria extrema- 
mente importante comparar, quantificando, a eficiência energética deste método de aquecimento relativamente ao $\mathrm{AC}$ e ao aquecimento com MO. Tendo em conta algumas evidências experimentais, nomeadamente as diferenças observadas no rendimento, tempo de reação e seletividade de algumas reações em comparação com o AC e MO, seria importante avaliar a existência de efeitos eletroquímicos específicos do $\mathrm{AO}$ devido à perturbação elétrica existente no meio reacional e como é que esses efeitos afetam as reações químicas. Prevê-se ainda a elaboração de estudos que permitam clarificar a influência do efeito do campo elétrico nas propriedades de transferência de massa, de forma a otimizar futuras aplicações do AO. Por último, seria importante expandir a utilização deste método de aquecimento aplicando-o a outras áreas da química, como por exemplo, na química dos materiais, na síntese inorgânica, na síntese de polímeros e na extração de produtos naturais. Este método de aquecimento tem um grande potencial de aplicação na indústria, particularmente à medida que for sendo aplicado a outras sínteses usando água como solvente, devido à elevada capacidade térmica, o baixo custo e a natureza não tóxica deste solvente, ou de outros solventes considerados mais benignos para o ambiente.

\section{AgRAdECIMENTOS}

Os autores agradecem à Universidade de Aveiro e à Fundação para a Ciência e a Tecnologia (FCT)/Ministério da Educação e Ciência pelo apoio financeiro ao projeto FCOMP01-0124FEDER-010840-PTDC/QUI-QUI/102454/2008 e às unidades de investigação QOPNA (FCT UID/ QUI/00062/2013), CIQUP (Pest-C/QUI/UI0081/2013) e REQUIMTE (UID/MULTI/04378/2013 - POCI/01/0145/ FERDER/007728), as quais são cofinanciadas pelo FEDER no âmbito do Acordo de Parceria PT2020. Vera L. M. Silva agradece à FCT a bolsa de pós-doutoramento SFRH/ BPD/108807/2015.

\section{REFERÊNCIAS}

[1] B.E. Getchel, Agr. Eng. 16 (1935) 408-410.

[2] S.V. McConnell, P.O. Robert, Wiener vending machine. US patent 2139690 (1938).

[3] A. Schade, Prevention of enzymatic discoloration of potatoes. US patent, 2, 569, 075; 1951.

[4] S. Mizrahi, I. Kopelman, J. Perlaman, J. Food Technol. 10 (1975) 281-288.

[5] P.J. Skudder, Food Eng. 60 (1988) 99-101.

[6] A.M.G. Silva, V.L.M. Silva, C. Queirós, J. Pinto, Boletim da Sociedade Portuguesa de Química 125 (2012) 61-68.

[7] S. Ceylan, L. Coutable, J. Wegner, A. Kirschning, Chem. Eur. J. 17 (2011) 1884-1893.

[8] P.J. Fryer, Z. Li, J. Food Sci. Technol. 4 (1993) 364-369.

[9] M. Lima, B.F. Heskett, S.K. Sastry, J. Food Process Eng. 22 (1999) 41-54.

[10] M. C. Knirsch, Tese de Mestrado, Universidade de São Paulo, Faculdade de Ciências Farmacêuticas (São Paulo, Brasil), 2010.

[11] N. Kaur, A.K. Singh, Crit. Rev. Food Sci. Nutr. 56 (2016)
2338-2351.

[12] R. Porta, M. Benaglia, A. Puglisi, Org. Process Res. Dev. 20 (2016) 2-25.

[13] S.K. Sastry, Novel Food Processing Technologies, Ed. Gustavo V. Barbosa-Canóvas, Maria S. Tapia, M. Pilar Cano, CRC Press, Boce Raton, FL, 2005.

[14] A.A. Vicente, I. Castro, J.A. Teixeira, Ohmic Heating for Food Processing. In: Thermal Food Processing: Modelling, Quality Assurance and Innovations, CRC press, New York (ISBN 1-57444-628-2), 2006.

[15] I. Castro, J.A. Teixeira, S. Salengke, S.K. Sastry, A.A. Vicente, J. Food Process Eng. 26 (2003) 17-29.

[16] M. Lima, B.F. Heskitt, L.L. Burianek, S.E. Nokes, S.K. Sastry, J. Food Process. Preserv. 23 (1999) 421-443.

[17] K. Allen, V. Eidman, J. Kinsey, Food Tech. 50 (1996) 269273.

[18] S.K. Sastry, J. Food Process Eng. 15 (1992) 263-278.

[19] Scopus database (acedida em 28-10-2016).

[20] R.N. Pereira, R.M. Rodrigues, Z. Genisheva, H. Oliveira, V. de Freitas, J.A. Teixeira, A.A. Vicente, LWT- Food Sci. Tech. 74 (2016) 493-503.

[21] R.N. Pereira, R.M. Rodrigues, O.L. Ramos, F.X. Malcata, J.A. Teixeira, A.A. Vicente, Food Bioprocess Tech. 9 (2016) 576-587.

[22] R.M. Rodrigues, A.J. Martins, O.L. Ramos, F.X. Malcata, J.A. Teixeira, A.A. Vicente, R.N. Pereira, Food Hydrocoll. 43 (2015) 329-339.

[23] R.N. Pereira, J.A. Teixeira, A.A. Vicente, J. Agric. Food Chem. 59 (2011) 11589-11597.

[24] R.N. Pereira, B.W.S. Souza, M.A. Cerqueira, J.A. Teixeira, A.A. Vicente, Biomacromolecules 11 (2010) 2912-2918.

[25] M.C. Knirsch, C.A. Santos, A.A.M.O.S. Vicente, T.C.V. Penna, Trends Food Sci. Tech. 21 (2010) 436-441.

[26] B.W.S. Souza, M.A. Cerqueira, A. Casariego, A.M.P. Lima, J.A. Teixeira, A.A. Vicente, Food Hydrocoll. 23 (2009) 2110-2115.

[27] R.N. Pereira, R.C. Martins, A.A.Vicente, J. Dairy Sci. 91 (2008) 2925-2937.

[28] J. Pinto, V.L.M. Silva, A.M.G. Silva, A.M.S. Silva, J.C.S. Costa, L.M.N.B.F. Santos, R. Enes, J.A.S. Cavaleiro, A.A.M.O.S. Vicente, J.A.C. Teixeira, Green Chem. 15 (2013) 970-975.

[29] V.L.M. Silva, A.M.S. Silva, L.M.N.B.F. Santos, A.M.G. Silva, J. Pinto; R. Enes, J.A.S. Cavaleiro, A.A.M.O.S. Vicente, J.A.C. Teixeira, A. Morais, J.C.S. Costa, Reator para síntese química com aquecimento óhmico, método e suas aplicações, Patente Portuguesa no. 105908, 2011-09-27.

[30] J. Pinto, V.L.M. Silva, A.M.G. Silva, L.M.N.B.F. Santos, A.M.S. Silva, J. Org. Chem. 80 (2015) 6649-6659.

[31] J. Pinto, V.L.M. Silva, L.M.N.B.F. Santos, A.M.S. Silva, Eur. J. Org. Chem. (2016) 2888-2896.

[32] M.F.C. Cardoso, A.T.P.C. Gomes, V.L.M. Silva, A.M.S. Silva, M.G.P.M.S. Neves, F.C.Silva, V.F. Ferreira, J.A.S. Cavaleiro, RSC Adv. 5 (2015) 66192-66199.

[33] R.G. Soengas, V.L.M. Silva, J. Pinto, H. Rodríguez-Solla, A.M.S. Silva, Eur. J. Org. Chem. (2016) 99-107. 


\section{Submit to}

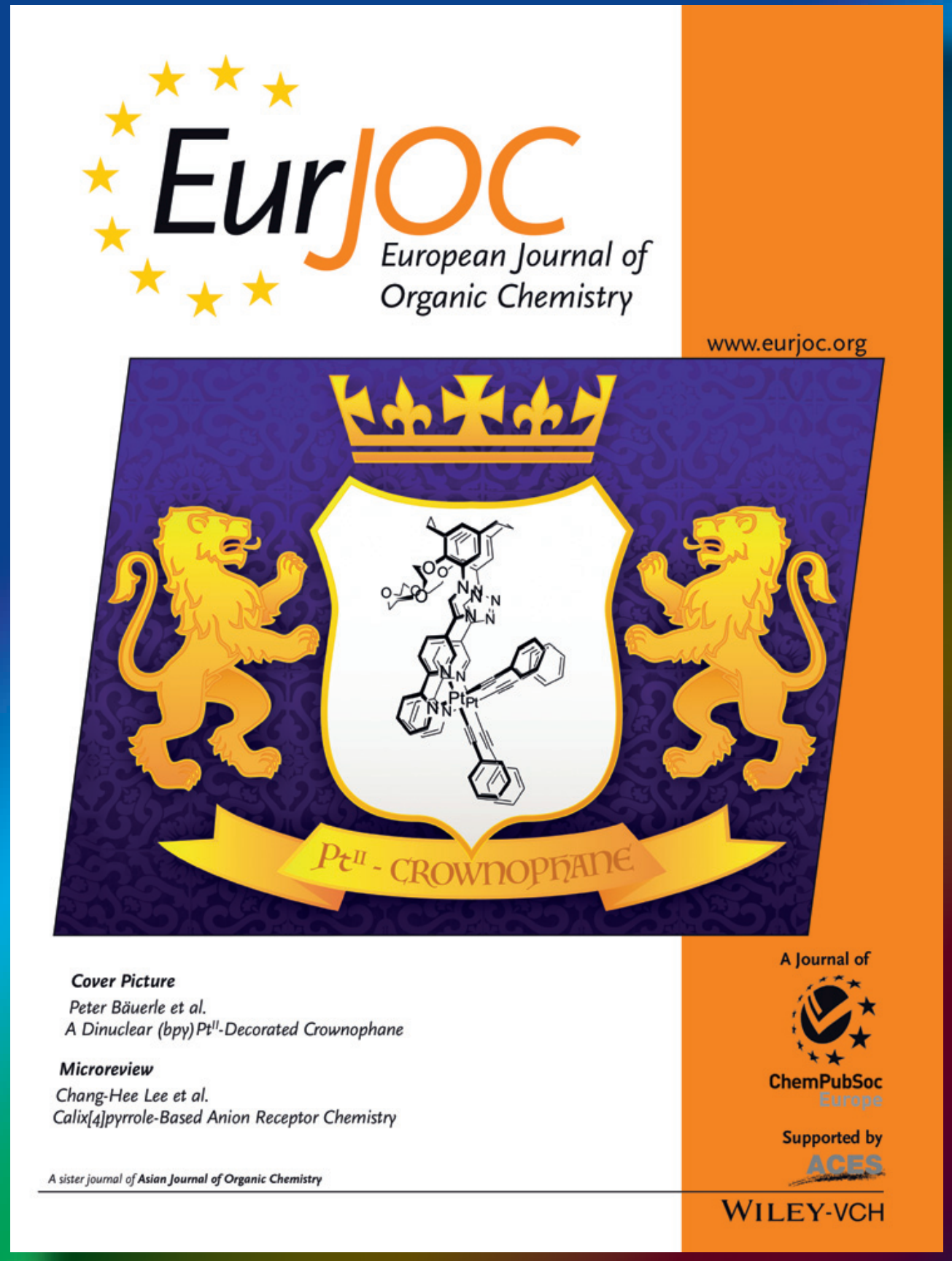

\section{www.eurjoc.org}

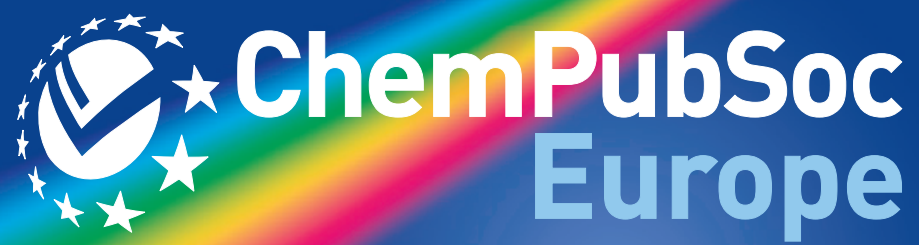

